\title{
Method for improving the accuracy of quality prediction in the manufacturing process of semiconductors
}

\author{
Shuichiro Chiba Hidekazu Yamanaka Tetsuroh Toyoshima \\ Production Automation Development Center, \\ Production Technology Development Group, SHARP Corporation \\ 2631-1, Ichinomoto-cho, Tenri, Nara 632-8567, Japan \\ E-mail: chiba.shuichiro@sharp.co.jp yamanaka.hidekazu@sharp.co.jp \\ toyoshima.tetsuroh@sharp.co.jp
}

\begin{abstract}
This paper describes the high accurate Virtual Metrology (VM) technique we propose, which may be applied to a mass production process of multiple products, e.g. semiconductors.

VM technology is necessary in a production process where sufficient amount of inspection data is not available. By applying VM technology, quality of the product and process error can be predicted based on the process data and relatively small amount of inspection data. Most common VM technology applied to these fields of manufacturing process for quality prediction uses a multivariate statistical model. However, the accuracy of prediction models using linear multiple regression drops when, during the generation of the model, a collinearity is found between terms in the process data that forms the explanatory variables. In addition, when updating the prediction model in order to increase its prediction accuracy, a collinearity among explanatory variables causes the regression coefficient to become unstable, resulting in a degradation of the prediction accuracy.

The method proposed in this paper enables us to create a quality prediction model composed of mutually uncorrelated variables and to perform prediction while updating the coefficient of each variable in the prediction model formula.

Our proposed technology will solve two problems: degradation of the quality prediction accuracy due to mutual interference between multiple process data and overfitting upon update of the metrology model.
\end{abstract}

\section{Introduction}

The semiconductor manufacturing process is comprised of various processing equipment used for the mass production of multiple products. Overall inspection is not implemented in such a production process due to faster takt times in the production line, cost, or other reasons. In many cases, sampling inspection is performed that does not allow sufficient inspection data to be obtained. In addition, the analysis of that sampling inspection may be time-consuming and impede the control of feedback of analysis results to the process.

In order to achieve efficient manufacturing of products that comply with specifications, VM (Virtual Metrology) is needed to predict quality based on the process data from the processing equipment. Current VM technology usually performs quality prediction by creating a prediction model for inspection results using linear multiple regression and forecasting product quality based on process data and the prediction model (Fig.1)[1].

\subsection{VM processing procedure}

VM technology can create a quality prediction model based on the process data obtained from the processing equipment in the production process and the inspection data obtained from the inspection equipment, and to predict the quality of the product to be processed next based on the created model and the new process data, thus enabling virtual 100\% inspection.

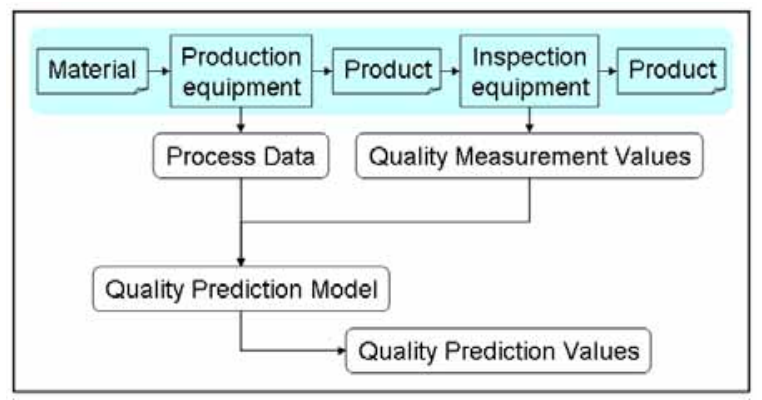

Figure 1 : Block Diagram - Virtual Metrology

It is also possible to minimize variations in each process and to prevent defective product by reflecting (feeding back) results of quality prediction derived through VM technology to the variables of processing equipment of the production line. 


\subsection{VM with a conventional method}

Conventional VM technologies usually performs quality prediction by creating a prediction model for inspection results using linear multiple regression, and forecasting the product quality based on process data and prediction model (Fig. 2). The below shows an example of a quality prediction method using conventional VM technology.

A prediction model that represents causal correlation is created based on the process data obtained from the processing equipment in the production process and the inspection data obtained from the inspection equipment. Below shows an example of a prediction model formula using linear multiple regression.

In this example, prediction model formula consisting of Multiple Linear Regression (MLR) as shown below is created by using an explanatory variable matrix. The matrix consist of $n$ lines $p$ columns $\left(\mathrm{X}_{11}, \mathrm{X}_{12}, \cdots, \mathrm{X}_{\mathrm{np}}\right.$ : number of the collected and accumulated process data) and the resulting output of this matrix, objective variables $\left(\mathrm{y}_{1}, \mathrm{y}_{2}, \cdots \mathrm{y}_{\mathrm{n}}\right.$ : inspection data).

$$
\left.\begin{array}{c}
y_{1}=a_{1} x_{11}+a_{2} x_{12}+\cdots+a_{p} x_{1 p} \\
y_{2}=a_{1} x_{21}+a_{2} x_{22}+\cdots+a_{p} x_{2 p} \\
\vdots \\
y_{n}=a_{1} x_{n 1}+a_{2} x_{n 2}+\cdots+a_{p} x_{n p}
\end{array}\right\}
$$

where, $\left\{a_{1}, a_{2}, \cdots, a_{p}\right\}$ is the partial regression coefficient. When the value of unknown parameter $a_{n}$ in the model formula of equation (1) is to be predicted by using least-square method, the following expression is used.

$$
\hat{Y}=a_{1} x_{1}+a_{2} x_{2}+\cdots+a_{n} x_{n}+K
$$

where, $X_{n}$ is the process data, $\hat{Y}$ is the prediction value of inspection data, and $K$ is the residual error between measurement value of inspection data and prediction value.

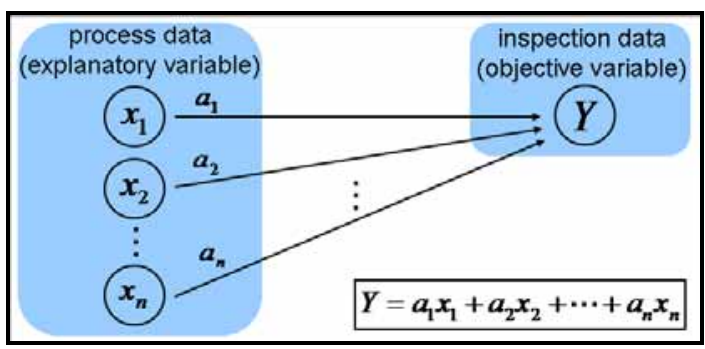

Figure 2 : Quality Prediction Model -Conventional Method (linear multiple regression)
Quality of the product is predicted by using the created prediction model formula of equation (2) and the new process data to be obtained from production equipment, achieving VM.

\subsection{VM related problems with the conventional method}

However, the accuracy of prediction models using linear multiple regression drops when, during the generation of the model, a collinearity is found between terms in the process data that forms the explanatory variables. In addition, when updating the prediction model in order to increase its prediction accuracy, a collinearity among explanatory variables causes the regression coefficient to become unstable, resulting in a degradation of the prediction accuracy.

Below describes the degradation of the prediction accuracy caused by a collinearity among explanatory variables.

Fig. 3 illustrates the conditions of model plane surface having a collinearity among explanatory variables. This means that a unique prediction model cannot be generated if a collinearity is found between variables.

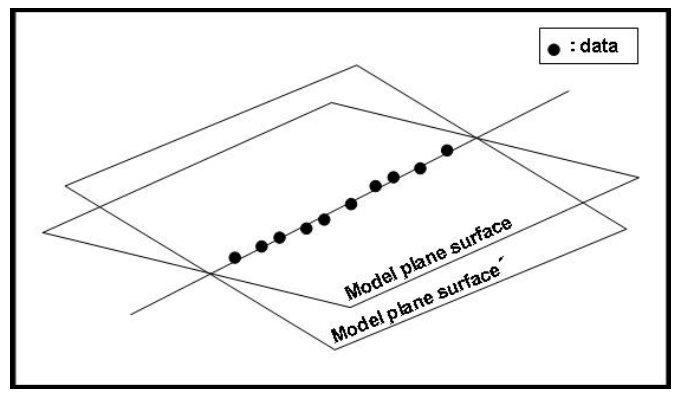

Figure 3 : Model plane surface having a collinearity among explanatory variables

As can be seen from Fig. 4, when unstable model having a collinearity among explanatory variables is updated by using newly entered data, the prediction model plane surface will greatly shift. Therefore, partial regression coefficient obtained by linear multiple regression becomes unstable and the model is now not a prediction model.

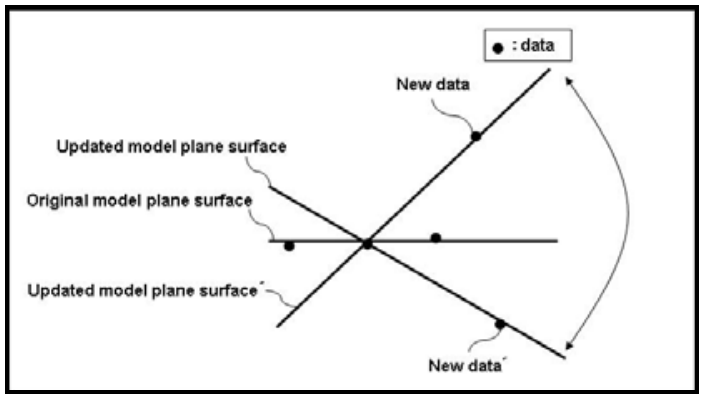

Figure 4 : Update of prediction model having a collinearity among explanatory variables 
Updating the regression coefficient of the quality prediction model, for example by using a Kalman filter [2], causes the regression coefficient to become unstable and degrades accuracy of the quality prediction if a collinearity is found between explanatory variables.

In contrast, when a collinearity is not found between explanatory variables, the model plane surface is kept stable even if the regression coefficient of the prediction model is updated for example by using the Kalman filter.

Therefore, to maintain the quality prediction accuracy, it is necessary to prepare a prediction model formula composed of mutually uncorrelated variables.

\section{Methodology (Model Architecture)}

We will express a prediction model formula composed of mutually uncorrelated variables based on process data and inspection results and will disclose a method that enables prediction to be performed while updating the coefficient of each variable in the prediction model formula (Fig.7, Fig.8).

\subsection{Structure of proposed method}

Our proposed method creates a VM model based on the process data obtained from the production equipment in the production process and the inspection data obtained from the inspection equipment, in the same way as with a conventional VM technology. The VM model for quality prediction is created using the modeling method with mutually uncorrelated variables and will be updated as new process data is acquired (Fig. 5).

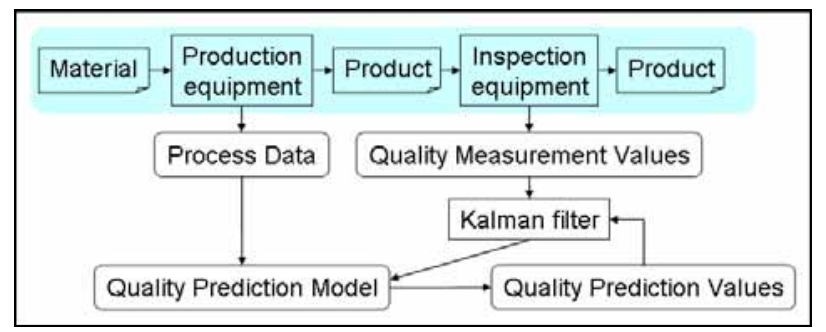

Figure 5 : Schematic Block Diagram

In this paper, Partial Least Squares (PLS) is used as the modeling method and a Kalman filter is used to update the quality prediction model.

Because the model employed for this proposed method has no collinearity between variables, model plane surface is kept stable as shown in Fig. 6.

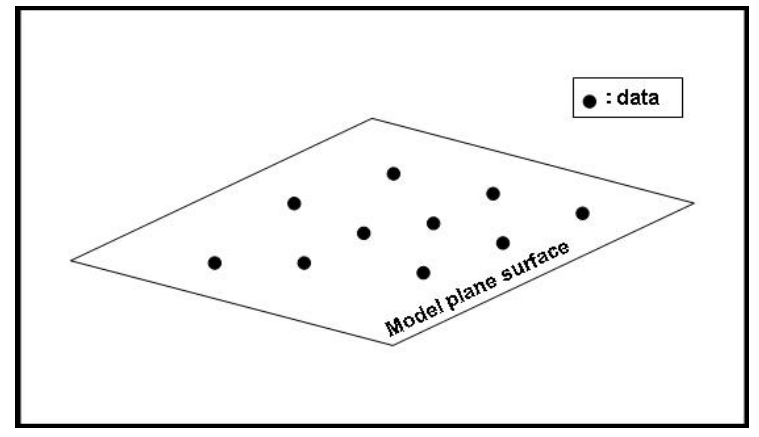

Figure 6 : Model plane surface having no collinearity between explanatory variables

As shown in Fig. 7, when a prediction model having no collinearity between explanatory variables is updated with data which deviates from the model plane surface, significant movement from the original prediction model will not occur.

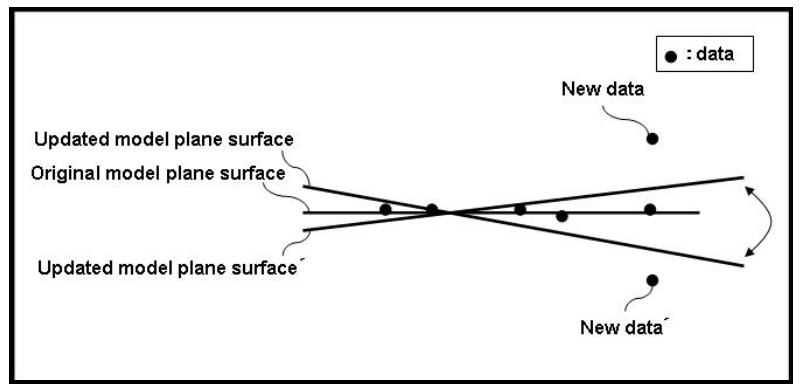

Figure 7 : Update of a prediction model having no collinearity between explanatory variables

\subsection{Processing procedure with proposed method}

\subsubsection{Creating PLS model}

An example of our proposed method is expressed in the prediction model formula (Fig. 8) of Equation (3) using the modeling method with mutually uncorrelated variables of Partial Least Squares (PLS).

$$
\hat{y}_{t}=q_{t 0} t_{t 0}+q_{t 1} t_{t 1}+\cdots+q_{t j} t_{t j}+K_{t}
$$

where, $\hat{y}_{t}$ is the quality prediction value (objective variable), $\left\{t_{t 0}, t_{t 1}, \cdots, t_{t j}\right\}$ is the mutually uncorrelated variables derived from process data measurement value (objective variable), $\left\{q_{t 0}, q_{t 1}, \cdots, q_{t j}\right\}$ is the coefficient of each variable, and $K_{t}$ is the bias term. 


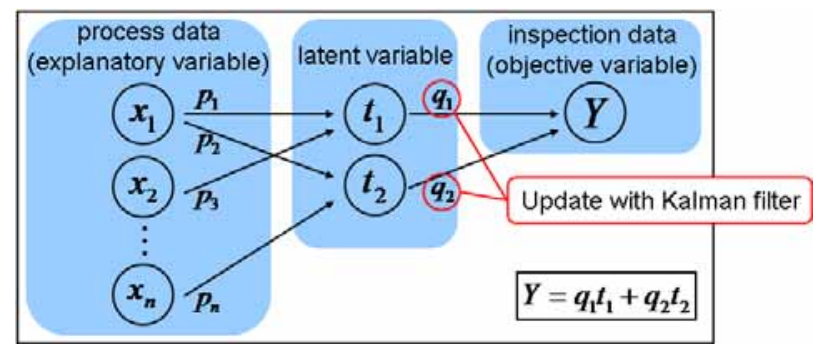

Figure 8:Quality Prediction Model and Update - Proposed Method (PLS)

\subsubsection{Update of model with Kalman filter}

Our proposed method includes updating the quality prediction model formula in order to maintain and improve prediction accuracy even in the event of aging of the processing equipment or a reduction in the sampling frequency of the quality inspection. As a method for updating the prediction formula, we will present a Kalman filter that greatly contributes to the improvement of the prediction accuracy with minimal calculations. This Kalman filter will be employed to update the values for the coefficients of each variable ( $\left.q_{t 0}, q_{t 1}, \cdots, q_{t j}\right)$ and the bias term $\left(K_{t}\right)$. The bias term Kt presents values such as difference value between units, which have been set during creation of the prediction model.

When the Kalman filter is used to modify coefficient of the prediction model formula, the following observation equation and prediction equation are prepared.

$$
\begin{aligned}
& y_{t}=H_{t} \alpha_{t}+v_{t} \\
& \alpha_{t+1}=A_{t} \alpha_{t}+w_{t}
\end{aligned}
$$

where, $y_{t}$ is the measurement value of inspection data (objective variable matrix), $H_{t}$ is the process data (explanatory variable matrix), $\alpha_{t}$ is the weight factor, $v_{t}$ is the observed noise which is assumed to be zero mean Gaussian white noise, $A_{t}$ is the control-input model which is applied to the weight factor, $v_{t}$, and $w_{t}$ is the process noise.

The process data $H_{t}$ and weight factor $\alpha_{t}$ are respectively expressed as follows:

$$
\begin{aligned}
& H_{t}=\left[\begin{array}{lll}
t_{t 0} t_{t 1} & \cdots & t_{t j}
\end{array}\right]-(6) \\
& \alpha_{t}=\left[\begin{array}{llll}
q_{t 0} & q_{t 1} & \cdots & q_{t j}
\end{array}\right]^{T}-(7)
\end{aligned}
$$

(“ $T$ ” in eq.(7) : transposed matrix)
Coefficients $\left(q_{t 0}, q_{t 1}, \cdots, q_{t j}\right)$ in the prediction model formula of Equation (6) and (7) are modified and the quality prediction values are calculated according to the following procedure.

Based on the inspection data measurement value and quality prediction value, new coefficients are derived using Kalman filter. The initial values of the coefficients are derived based on the process data and workmanship measurement value, while deriving the prediction model formula described above.

The quality prediction value will be derived from the newly acquired process data and according to the model formula.

$$
y_{t}=q_{t 0} t_{t 0}+q_{t 1} t_{t 1}+\cdots+q_{t j} t_{t j}+K_{t}
$$

where, $y_{t}$ is the quality prediction value (objective variable), $\left\{t_{1}, t_{2}, \cdots, t_{j}\right\}$ is mutually uncorrelated variables derived from process data measurement value (objective variable), and $\left\{q_{t 0}, q_{t 1}, \cdots, q_{t j}\right\}$ is the coefficient of these mutually uncorrelated variable.

With our proposed method, even if the process data shows a collinearity, the updating of the regression coefficient will be stabilized during the updating of the prediction model formula, enabling quality prediction with high accuracy to be achieved.

\section{Evaluation}

We have applied our proposed method to predict the film thickness during the deposition process for semiconductors, and observed an improvement of the prediction accuracy (prediction error) in comparison with conventional methods (Fig. 9, Table 1).

Fig. 9 is the time sequence diagram indicating prediction error resulting from the proposed method and conventional method. Values closer to "0[a.u.]" indicate smaller prediction error. It is clear that the proposed method provides higher prediction accuracy than the conventional ones.

Table 1 shows the calculated root mean square errors in prediction values (film thickness predicted by the proposed method and conventional method, respectively) with respect to the target film thickness value. Smaller values show smaller errors. Again, the proposed method has accurate prediction performance in comparison with the conventional method. 


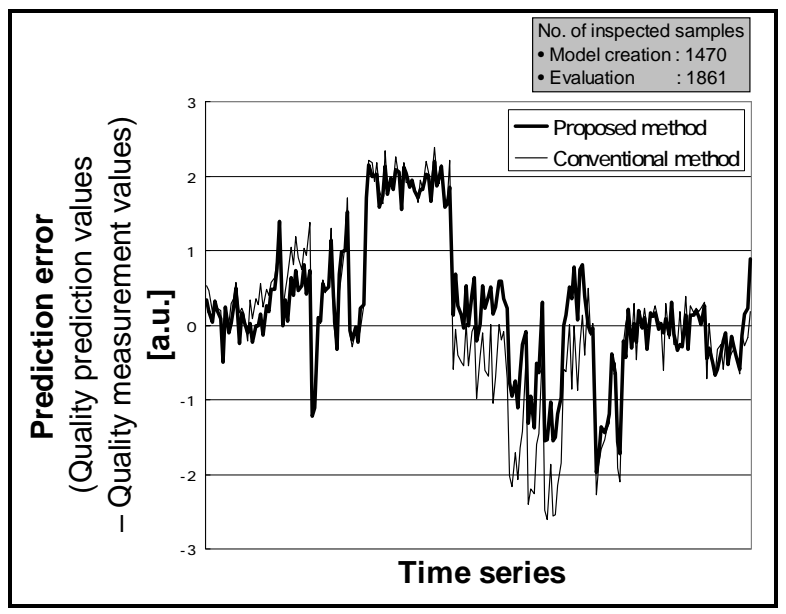

Figure9:Corroborative Results Graph

Table1:Corroborative Results (prediction error)

\begin{tabular}{|c|c|} 
& $\begin{array}{r}\text { Prediction value } \\
\text { RMS error } \\
\text { / desired value } \\
{[\%]}\end{array}$ \\
\hline $\begin{array}{c}\text { Proposed method } \\
\text { (PLS +Kalman Filter) }\end{array}$ & 1.15 \\
\hline $\begin{array}{c}\text { Conventional method } \\
\text { ( Multiple Linear Re- } \\
\text { gression } \\
\text { +Kalman Filter) }\end{array}$ & 1.31 \\
\hline
\end{tabular}

We further confirmed that an accurate prediction for the film thickness could be secured even when updating the prediction model using inspection results with a sampling of one out of 50 (Fig. 10).

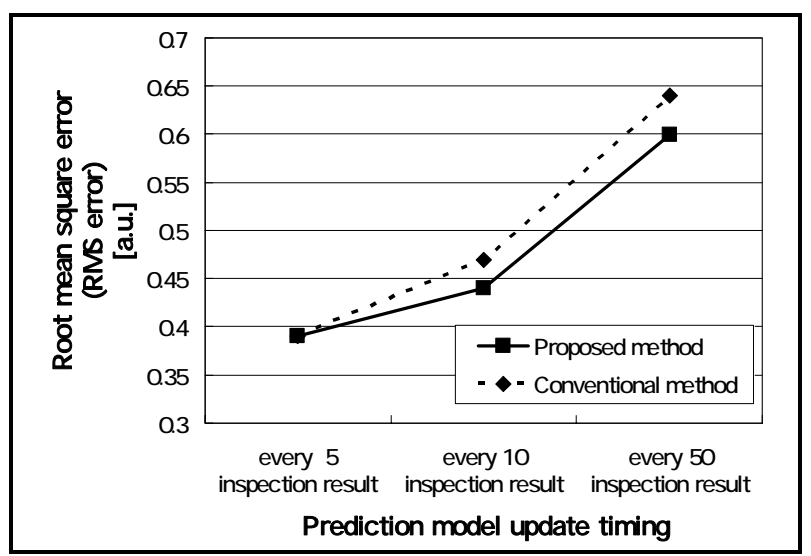

Figure 10:Corroborative Results Graph - Comparison of RMS error

\section{Conclusions}

We have expressed a prediction model formula composed of mutually uncorrelated variables and presented a method for updating that model formula. The adoption of our method enabled quality prediction of satisfactory accuracy to be achieved even in the case of a collinearity among process data terms. In addition, highly accurate quality prediction was also obtained for inspections with a small amount of sampling.

In the future, we will strive to secure high-accuracy quality prediction by creating a model that takes equipment maintenance into account as an important factor for product quality.

\section{References}

[1] Lynn, Shane and Ringwood, John and Ragnoli, Emanuele and McLoone, Sean and MacGearailt, Niall, "Virtual Metrology for Plasma Etch using Tool Variables," ASMC, pp.143-148, 2009.

[2] G. Welch, G. Bishop, An introduction to the Kalman Filter, UNC-Chapel Hill, TR 95-041, July 24, 2006. 